\title{
Highly Enantioselective Alkynylation of $\alpha$-Keto Ester: An Efficient Method for Constructing a Chiral Tertiary Carbon Center
}

Biao Jiang, ${ }^{*}$ Zili Chen and Xiaoxia Tang

Melting point were uncorrected. ${ }^{1} \mathrm{H}$ NMR spectra were recorded on $300 \mathrm{MHz}$ spectrometers with TMS as an internal standard and $\mathrm{CDCl}_{3}$ as solvent. ${ }^{13} \mathrm{C}$ NMR spectra were recorded on $75 \mathrm{MHz}$ spectrometers with TMS as an internal standard and $\mathrm{CDCl}_{3}$ as solvent. ${ }^{19}$ FNMR spectra were recorded on $282 \mathrm{MHz}$ spectrometer with $\mathrm{CDCl}_{3}$ as solvent. Coupling constants, $J$ values, were given in Hz. IR spectra were taken on a Shimadzu 440-IR spectrophotometer. MS spectra were run respectively on a finnigan 4021 GC MS/DC and Varian MAT 21 instrument with an ionizing voltage of $70 \mathrm{eV}$.

\section{Preparation of $(1 S, 2 S)$-2- $N, N$-dimethylamino-3-( $p$-nitrophenyl)propane- 1,3-diol:}

(1S,2S)-2-amino-3-(p-nitrophenyl)propane-1,3-diol (1) (5 g, $23.6 \mathrm{mmol})$, aqueous HCHO $(37-40 \%, 7.5 \mathrm{~mL})$ and $\mathrm{HCOOH}(98 \%, 10 \mathrm{~mL})$ was added to a $25 \mathrm{~mL}$ flask and refluxed for $8 \mathrm{~h}$. After removal of the solvent under reduced pressure, the residue was neutralized with $1 \mathrm{~N} \mathrm{NaOH}(30 \mathrm{~mL})$ and extracted with $\mathrm{CH}_{2} \mathrm{Cl}_{2}(3 \times 30 \mathrm{~mL})$. The combined organic phase was washed with brine, dried $\left(\mathrm{Na}_{2} \mathrm{SO}_{4}\right)$ and filtered. After removed of the solvent, the residue was subjected to flash chromatography on a short basic $\mathrm{Al}_{2} \mathrm{O}_{3}$ column (eluted with $\left.\mathrm{CH}_{2} \mathrm{Cl}_{2} / \mathrm{CH}_{3} \mathrm{OH}=10: 1\right)$ to give product (5.6 g, 99\%) as a brown solid: mp 88.8-89.1 ${ }^{\circ} \mathrm{C} ;[\alpha]_{\mathrm{D}}^{20}=+25.7\left(c, 0.505, \mathrm{CH}_{3} \mathrm{OH}\right) ;{ }^{1} \mathrm{H}$ NMR $\left(300 \mathrm{MHz}, \mathrm{CDCl}_{3}\right) \delta_{\mathrm{H}} 8.20(\mathrm{~d}, J=7.8 \mathrm{~Hz}$, 2H), $7.58(\mathrm{~d}, J=7.8 \mathrm{~Hz}, 2 \mathrm{H}), 4.55(\mathrm{~d}, J=9.6 \mathrm{~Hz}, 1 \mathrm{H}), 3.60(\mathrm{~d}, J=4.5 \mathrm{~Hz}, 2 \mathrm{H}), 2.60-2.55$ 
$(\mathrm{m}, 1 \mathrm{H}), 2.53(\mathrm{~s}, 6 \mathrm{H}) ;{ }^{13} \mathrm{C} \mathrm{NMR}\left(75 \mathrm{MHz}, \mathrm{CDCl}_{3}\right) \delta_{\mathrm{C}} 150.0,147.0,127.7,123.2,70.8$, 69.6, 57.1, 41.2 ppm; IR (neat) 3366, 3069, 2980, 2918, 1694, 1599, 1525, 1352, 1199, 1061, 998, 858, 832, 748, 698; Ms m/e (relative intensity) $240\left(\mathrm{M}^{+}, 8.15\right), 200$ (2.90), 195 (7.07), 153 (5.34), 105 (21.56), 88 (85.87), 58 (48.55), 42 (100.00); Anal. calcd. for $\mathrm{C}_{11} \mathrm{H}_{16} \mathrm{~N}_{2} \mathrm{O}_{4}$ : C, 54.99; H, 6.71; N, 11.66. found: C, 54.92; H, 6.81\%; N, 11.35.

\section{Preparation of $(1 S, 2 S)-3$-( $t$-butyldimethylsilyloxy)-2- $N, N$-dimethylamino-1-( $p$-nitro phenyl)propane-1-ol (1):}

A solution of above substrate $(1.95 \mathrm{~g}, 8.1 \mathrm{mmol})$ in $\mathrm{CH}_{2} \mathrm{Cl}_{2}(30 \mathrm{~mL})$ was treated with tert-butyldimethysilylchloride $(1.28 \mathrm{~g}, 8.5 \mathrm{mmol})$, imidazole $(1.4 \mathrm{~g}, 20.6 \mathrm{mmol})$ and a catalytic amount of DMAP (10 mg) overnight at $\mathrm{rt}$ under $\mathrm{N}_{2}$. The reaction mixture was poured into water $(20 \mathrm{~mL})$ and then neutralized with cold aqueous $\mathrm{HCl}(0.5 \mathrm{M})$ to $\mathrm{pH}=8$. The aqueous phase was extracted with $\mathrm{CH}_{2} \mathrm{Cl}_{2}(2 \times 10 \mathrm{~mL})$. The combined organic phase was washed with saturated $\mathrm{Na}_{2} \mathrm{CO}_{3}$ solution, brine, dried with anhydrous $\mathrm{Na}_{2} \mathrm{SO}_{4}$ and filtered. After removal of solvent, the residue was purified by flash chromatography on silica gel column (eluted with $\left.\mathrm{CH}_{2} \mathrm{Cl}_{2} / \mathrm{CH}_{3} \mathrm{OH}=20: 1\right)$ to give $\mathbf{1}(2.72 \mathrm{~g}, 95 \%)$ as a brown oil: $[\alpha]_{\mathrm{D}}{ }^{20}=-15.8\left(c, 1.09, \mathrm{CHCl}_{3}\right) ;{ }^{1} \mathrm{H}$ NMR $\left(300 \mathrm{MHz}, \mathrm{CDCl}_{3}\right) \delta_{\mathrm{H}} 8.25-8.20(\mathrm{~d}, J=8.5$ $\mathrm{Hz}, 2 \mathrm{H}), 7.60-7.55(\mathrm{~d}, J=8.5 \mathrm{~Hz}, 2 \mathrm{H}), 4.65(\mathrm{~d}, J=9.7 \mathrm{~Hz}, 1 \mathrm{H}), 3.7-3.6(\mathrm{dd}, J=11.3 \mathrm{~Hz}$, $2.7 \mathrm{~Hz}, 1 \mathrm{H}), 3.5-3.45$ (dd, $J=11.3 \mathrm{~Hz}, 6 \mathrm{~Hz}, 1 \mathrm{H}), 2.5$ (m, 7H), 1.85 (s, 9H), 0.1 (s, 6H).

${ }^{13} \mathrm{C}$ NMR $\left(75 \mathrm{MHz}, \mathrm{CDCl}_{3}\right) \delta_{\mathrm{H}} 150.2,147.4,128.0,123.3,71.3,69.0,57.1,41.6,25.7$, 17.9, -5.9 ppm; IR (neat) 3344, 2954, 2931, 2858, 1606, 1525, 1471, 1349, 1257, 1114, 1039, 946, 839, 777, 699; Ms m/e (relative intensity) 297 ( $\left.\mathrm{M}^{+}-57,0.27\right), 209$ (8.18), 203 (17.45), 202 (100.00), 163 (2.33), 129 (1.84), 73 (27.37); Anal. calcd. for $\mathrm{C}_{17} \mathrm{H}_{30} \mathrm{~N}_{2} \mathrm{O}_{4} \mathrm{Si}$ : C, $57.60 \% ; \mathrm{H}, 8.53 \% ; \mathrm{N}, 7.90 \%$. found: C, $57.82 \% ; \mathrm{H}, 8.18 \% ; \mathrm{N}, 7.77 \%$.

General procedure for the catalytic asymmetric alkynylation addition of $\alpha$-keto ester: To a solution of $\mathrm{Zn}(\mathrm{OTf})_{2}(0.2$ equiv., $0.1 \mathrm{mmol})$ and chiral ligand $(1 S, 2 S)-\mathbf{1}$ (0.22 equiv., $0.11 \mathrm{mmol}$ ) in terminal acetylene ( 3 equiv., $1.5 \mathrm{mmol}$ ) was added triethylamine ( 0.3 equiv., $0.15 \mathrm{mmol}$ ) under nitrogen atmosphere at ambient temperature for $2 \mathrm{~h}$. Then the $\alpha$-keto 
ester ( 1 equiv., $0.5 \mathrm{mmol}$ ) was introduced by syringe. The reaction mixture was stirred for 2 days at $70{ }^{\circ} \mathrm{C}$. The mixture was diluted petroleum ether $(20 \mathrm{~mL})$ and washed with $\mathrm{HCl}$ ( $0.5 \mathrm{M}, 3 \times 10 \mathrm{~mL}$, keeping $\mathrm{pH} \geq 4)$. The organic phase was washed with brine, distilled water, and dried with anhydrous $\mathrm{Na}_{2} \mathrm{SO}_{4}$. After removal of solvent, the crude product was purified through a short flash chromatograph (Petroleum ether/EtOAc=7/1) to yield the corresponding hydroxyl ynyl ester. The aqueous phase was neutralized with $\mathrm{NH}_{4} \mathrm{OH}$ to $\mathrm{pH}=8$ and extracted with $\mathrm{CH}_{2} \mathrm{Cl}_{2}(3 \times 10 \mathrm{~mL})$. The combined organic phase was washed with saturated aqueous $\mathrm{Na}_{2} \mathrm{CO}_{3}$, brine and distilled water, dried with anhydrous $\mathrm{Na}_{2} \mathrm{SO}_{4}$. After removal of solvent, the ligand was recovered in $98 \%$.

\section{Mehtyl (+)-2-hydroxyl-2,4-diphenyl-3-yn-butyrate (4a):}

$\mathrm{Zn}(\mathrm{OTf})_{2}(0.2$ eq. $)$ as additive, $(1 S, 2 S)-\mathbf{1}$ as ligand: Isolated in $91 \%$ yield and $89 \%$ ee (the reaction scale: Alkyne 3a is $1.5 \mathrm{mmol}$, the $\alpha$-keto ester $\mathbf{2 a}$ is $0.5 \mathrm{mmol}$ ) as determined by HPLC analysis (Chiralcel AD, hexane / $i-\mathrm{PrOH}=97 / 3,254 \mathrm{~nm}$ ) $\mathrm{t}_{\mathrm{r}} 24.020$ (major), 32.793 (minor); $\mathrm{Zn}(\mathrm{ODf})_{2}(0.2$ eq.) as additive, $(1 S, 2 S)-\mathbf{1}$ as ligand: Isolated in $83 \%$ yield and $92 \%$ ee (the reaction scale: Alkyne $3 \mathbf{a}$ is $1.5 \mathrm{mmol}$, the $\alpha$-keto ester $\mathbf{2 a}$ is $0.5 \mathrm{mmol}$ ) as determined by HPLC analysis (Chiralcel OD, $i$-PrOH / hexane = 97/3, $254 \mathrm{~nm}$ ) $t_{\mathrm{r}} 32.343$ (major), 44.263 (minor); $\mathrm{Zn}(\mathrm{OTf})_{2}$ (0.2 eq.) as additive, (+)- $N$-methylephedrine 5 as ligand: Isolated in $87 \%$ yield and $88 \%$ ee (the reaction scale: Alkyne 3a is $1.5 \mathrm{mmol}$, the $\alpha$-keto ester $\mathbf{2 a}$ is $0.5 \mathrm{mmol}$ ) as determined by HPLC analysis (Chiralcel OD, $i$-PrOH / hexane = 9/1, $254 \mathrm{~nm}$ ) $\mathrm{t}_{\mathrm{r}} 32.757$ (minor), 44.730 (major); $[\alpha]_{\mathrm{D}}{ }^{20}=+19.56\left(c, 4.04, \mathrm{CHCl}_{3}\right.$ ); IR (neat) 3493, 2953, 2227, 1738, 1599, 1491, 1450, 1430, 1259, 1185, 1175, 1097, 1072, 965, 766, $695 \mathrm{~cm}^{-1} ;{ }^{1} \mathrm{H}$ NMR $\left(\mathrm{CDCl}_{3}, 300 \mathrm{MHz}\right) \delta_{\mathrm{H}} 7.79(\mathrm{~m}, 2 \mathrm{H}), 7.55(\mathrm{~m}, 2 \mathrm{H}), 7.40(\mathrm{~m}, 6 \mathrm{H}), 4.40$ (s, $1 \mathrm{H}), 3.80(\mathrm{~s}, 3 \mathrm{H}) \mathrm{ppm} .{ }^{13} \mathrm{C} \mathrm{NMR}\left(\mathrm{CDCl}_{3}, 75 \mathrm{~Hz}\right) \delta_{\mathrm{C}} 172.7,139.5,132.2,129.2,129.1$, 128.7, 128.6, 126.5, 122.1, 87.2, 86.6, 73.5, 54.5 ppm; Ms m/e (relative intensity) 266 ( $\mathrm{M}^{+}$, 0.13), $250\left(\mathrm{M}^{+}-16,8.25\right), 207$ (100.00), 178 (7.71), 129 (65.73), 105 (24.47), 77 (18.20); HRMS for $\mathrm{C}_{17} \mathrm{H}_{14} \mathrm{O}_{3}$ : 266.0947; found: 266.0945 .

\section{Ethyl (+)-2-hydroxyl-2-sulfene-4-phenyl-3-yn-butyrate (4b):}


$\mathrm{Zn}(\mathrm{OTf})_{2}(0.2$ eq. $)$ as additive, $(1 S, 2 S)-\mathbf{1}$ as ligand: Isolated in $93 \%$ yield and $73 \%$ ee (the reaction scale: Alkyne $\mathbf{3 a}$ is $1.5 \mathrm{mmol}$, the $\alpha$-keto ester $\mathbf{2 b}$ is $0.5 \mathrm{mmol}$ ) as determined by HPLC analysis (Chiralcel AD, hexane / $i-\mathrm{PrOH}=85 / 15,254 \mathrm{~nm}$ ) $\mathrm{t}_{\mathrm{r}} 16.09$ (major), 19.30 (minor); $[\alpha]_{\mathrm{D}}^{20}=+11.08\left(c, 3.91, \mathrm{CHCl}_{3}\right)$; IR (neat) 3473, 2983, 2232, 1737, 1490, 1444, 1368, 1249, 1233, 1159, 1094, 1043, 855, 759, 707, $691 \mathrm{~cm}^{-1} ;{ }^{1} \mathrm{H}_{\mathrm{NMR}}\left(\mathrm{CDCl}_{3}, 300\right.$ MHz) $\delta_{\mathrm{H}} 7.50(\mathrm{~m}, 2 \mathrm{H}), 7.35-7.30(\mathrm{~m}, 5 \mathrm{H}), 6.95(\mathrm{~m}, 1 \mathrm{H}), 4.45(\mathrm{br}, 1 \mathrm{H}), 4.35(\mathrm{~m}, 2 \mathrm{H}), 1.25$ $(\mathrm{m}, 3 \mathrm{H}) \mathrm{ppm} ;{ }^{13} \mathrm{C} \mathrm{NMR}\left(\mathrm{CDCl}_{3}, 75 \mathrm{~Hz}\right) \delta_{\mathrm{C}} 170.9,143.7,131.9,129.1,128.3,126.9,126.3$, 126.2, 121.6, 86.7, 85.4, 70.5, 63.9, 13.9 ppm; Ms m/e (relative intensity) $286\left(\mathrm{M}^{+}, 0.12\right)$, 213 (100.00), 184 (3.66), 129 (45.10), 111 (33.38); HRMS for $\mathrm{C}_{16} \mathrm{H}_{14} \mathrm{SO}_{3}$ : 286.0657; found: 286.0660 .

\section{Methyl (+)-2-hydroxyl-2,6-diphenyl-3-yn-hexyrate (4c):}

$\mathrm{Zn}(\mathrm{OTf})_{2}(0.2$ eq.) as additive, $(1 S, 2 S)-\mathbf{1}$ as ligand: Isolated in $88 \%$ yield and $94 \%$ ee (the reaction scale: Alkyne $\mathbf{3 b}$ is $1.5 \mathrm{mmol}$, the $\alpha$-keto ester $\mathbf{2 a}$ is $0.5 \mathrm{mmol}$ ) as determined by HPLC analysis (Chiralcel AD, hexane / $i-\mathrm{PrOH}=95 / 5,254 \mathrm{~nm}$ ) $\mathrm{t}_{\mathrm{r}} 33.58$ (minor), 37.64 (major); $[\alpha]_{\mathrm{D}}{ }^{20}=+26.65\left(c, 4.83, \mathrm{CHCl}_{3}\right)$; IR (neat) 3487, 3030, 2954, 2853, 2243, $1737,1602,1450,1434,1255,1147,1082,1074,932,759,699 \mathrm{~cm}^{-1} ;{ }^{1} \mathrm{H}$ NMR $\left(\mathrm{CDCl}_{3}\right) \delta_{\mathrm{H}}$ $7.60(\mathrm{~m}, 2 \mathrm{H}), 7.35-7.20(\mathrm{~m}, 5 \mathrm{H}), 4.18(\mathrm{~s}, 1 \mathrm{H}), 3.75(\mathrm{~s}, 3 \mathrm{H}), 2.90$ (t, J=7.2 Hz, 2H), 2.65 (t, $J=7.2 \mathrm{~Hz}, 2 \mathrm{H}) \mathrm{ppm} ;{ }^{13} \mathrm{C} \mathrm{NMR}\left(\mathrm{CDCl}_{3}, 75 \mathrm{~Hz}\right) \delta_{\mathrm{C}} 172.9,140.6,139.7,128.8,128.7$, 128.6, 126.6, 126.5, 87.0, 79.3, 73.1, 54.4, 34.8, 21.3 ppm; Ms m/e (relative intensity): 235 ( $\mathrm{M}^{+}$- 59, 76.36), 217 (3.21), 202 (3.65), 157 (3.23), 144 (2.56), 129 (4.32), 105 (100.00), 91 (48.44); HRMS for $\mathrm{C}_{17} \mathrm{H}_{15} \mathrm{O}$ : 235.1085; found: 235.1104.

\section{Methyl (+)-2-hydroxyl-2-phenyl-5-'butyldimethylsilyloxyl-3-yn-pentyrate (4d):}

$\mathrm{Zn}(\mathrm{OTf})_{2}(0.2$ eq.) as additive, $(1 S, 2 S)-\mathbf{1}$ as ligand: Isolated in $83 \%$ yield and $91 \%$ ee (the reaction scale: Alkyne $\mathbf{3 c}$ is $1.5 \mathrm{mmol}$, the $\alpha$-keto ester $\mathbf{2 a}$ is $0.5 \mathrm{mmol}$ ) as determined by HPLC analysis (Chiralcel AD, hexane / $i-\mathrm{PrOH}=8 / 2,254 \mathrm{~nm}$ ) t 7.85 (minor), 8.53 (major); $[\alpha]_{\mathrm{D}}^{20}=+23.5\left(c, 0.71, \mathrm{CHCl}_{3}\right)$; IR (neat) 3495, 2957, 2931, 2859, 1741, 1452, $1257,1146,1095,1067,837,780,697 \mathrm{~cm}^{-1} ;{ }^{1} \mathrm{H}$ NMR $\left(\mathrm{CDCl}_{3}, 300 \mathrm{MHz}\right) \delta_{\mathrm{H}} 7.60(\mathrm{~m}, 2 \mathrm{H})$, 
$7.29(\mathrm{~m}, 3 \mathrm{H}), 4.39$ (s, 2H), $4.18(\mathrm{~s}, 1 \mathrm{H}), 3.70$ (s, 3H), 0.85 (s, 9H), $0.06(\mathrm{~s}, 6 \mathrm{H}) \mathrm{ppm} ;{ }^{13} \mathrm{C}$ $\operatorname{NMR}\left(\mathrm{CDCl}_{3}, 75 \mathrm{~Hz}\right) \delta_{\mathrm{C}} 172.5,139.2,128.9,128.5,126.5,126.4,85.4,82.8,72.9,54.4$, 52.0, 26.0, 18.5, -4.9 ppm; Ms m/e (relative intensity) $334\left(\mathrm{M}^{+}, 0.35\right), 318$ (100.00), 276 (22.22), 246 (45.05), 217 (19.49), 189 (23.75), 171 (32.83), 115 (36.90), 75 (42.68); HRMS for $\mathrm{C}_{17} \mathrm{H}_{23} \mathrm{SiO}_{4}$ : 319.1354; found: 319.1360 .

\section{Methyl (+)-2-hydroxyl-2-[( $N$-'butoxylcarbonyl)-3-indole]-4-phenyl-3-yn-butyrate} (4e):

$\mathrm{Zn}(\mathrm{OTf})_{2}(0.2$ eq.) as additive, $(1 S, 2 S)-\mathbf{1}$ as ligand: Isolated in $81 \%$ yield and $83 \%$ ee (the reaction scale: Alkyne $3 \mathbf{a}$ is $2.5 \mathrm{mmol}$, the $\alpha$-keto ester $\mathbf{2 c}$ is $0.5 \mathrm{mmol}$ ) as determined by HPLC analysis (Chiralcel AD, hexane / $i-\mathrm{PrOH}=7 / 3,254 \mathrm{~nm}$ ) $\mathrm{t}_{\mathrm{r}} 10.240$ (minor), 14.593 (major); $[\alpha]_{\mathrm{D}}{ }^{20}=+8.93\left(c, 3.54, \mathrm{CHCl}_{3}\right)$; IR (neat) 3474, 2928, 1739, 1453, 1373, $1255,1156,1080,751,691 \mathrm{~cm}^{-1} ;{ }^{1} \mathrm{H} \mathrm{NMR}\left(\mathrm{CDCl}_{3}, 300 \mathrm{MHz}\right) \delta_{\mathrm{H}} 8.20(\mathrm{~d}, J=8.0 \mathrm{~Hz}, 1 \mathrm{H})$, $7.95(\mathrm{~s}, 1 \mathrm{H}), 7.80(\mathrm{~d}, J=7.5 \mathrm{~Hz}, 1 \mathrm{H}), 7.52(\mathrm{~m}, 2 \mathrm{H}), 7.35(\mathrm{~m}, 4 \mathrm{H}), 7.25(\mathrm{~m}, 1 \mathrm{H}), 4.30(\mathrm{br}$, 1H), $3.80(\mathrm{~s}, 3 \mathrm{H}), 1.72(\mathrm{~s}, 9 \mathrm{H}) \mathrm{ppm} ;{ }^{13} \mathrm{C} \mathrm{NMR}\left(\mathrm{CDCl}_{3}, 75 \mathrm{~Hz}\right) \delta_{\mathrm{C}} 172.2,149.7,136.4$, 132.2, 129.3, 128.6, 127.4, 125.7, 124.9, 123.2, 122.0, 120.7, 119.8, 115.6, 86.5, 86.0, 84.4, 69.3, 54.6, 28.4 ppm; Ms m/e (relative intensity) $389\left(\mathrm{M}^{+}-16,1.21\right), 347$ (21.99), 333 (13.26), 291 (100.00), 247 (63.27), 206 (16.48), 144 (17.05), 129 (55.79), 57 (68.39); HRMS for $\mathrm{C}_{24} \mathrm{H}_{23} \mathrm{NO}_{5}$ : 405.1562; found: 405.1569 .

\section{Methyl (+)-2-hydroxyl-2-[( $N-{ }^{t}$ butoxylcarbonyl)-3-indole]-6-phenyl-3-yn-hexyrate} (4f):

$\mathrm{Zn}(\mathrm{OTf})_{2}(0.2$ eq.) as additive, $(1 S, 2 S)-\mathbf{1}$ as ligand: Isolated in $76 \%$ yield and $86 \%$ ee (the reaction scale: Alkyne $\mathbf{3 b}$ is $2.5 \mathrm{mmol}$, the $\alpha$-keto ester $\mathbf{2 c}$ is $0.5 \mathrm{mmol}$ ) as determined by HPLC analysis (Chiralcel AD, hexane / $i-\mathrm{PrOH}=75 / 25,254 \mathrm{~nm}$ ) $\mathrm{t}_{\mathrm{r}} 13.71$ (minor), 15.64 (major); $[\alpha]_{\mathrm{D}}{ }^{20}=+14.4\left(c, 1.265, \mathrm{CHCl}_{3}\right)$; IR (neat) 3487, 2979, 2930, 2239, 1737, $1454,1374,1257,1157,1089,1060,1021,749,700 \mathrm{~cm}^{-1} ;{ }^{1} \mathrm{H}$ NMR $\left(\mathrm{CDCl}_{3}\right) \delta_{\mathrm{H}} 8.15(\mathrm{~d}, J$ $=8.1 \mathrm{~Hz}, 1 \mathrm{H}), 7.85(\mathrm{~s}, 1 \mathrm{H}), 7.65(\mathrm{~d}, J=7.9 \mathrm{~Hz}, 1 \mathrm{H}), 7.35-7.20(\mathrm{~m}, 7 \mathrm{H}), 4.05(\mathrm{br}, 1 \mathrm{H})$, $3.80(\mathrm{~s}, 3 \mathrm{H}), 2.90(\mathrm{t}, J=7.6 \mathrm{~Hz}, 2 \mathrm{H}), 2.60(\mathrm{t}, J=7.6 \mathrm{~Hz}, 2 \mathrm{H}), 1.65(\mathrm{~s}, 9 \mathrm{H}) \mathrm{ppm} ;{ }^{13} \mathrm{C} \mathrm{NMR}$ 
$\left(\mathrm{CDCl}_{3}, 75 \mathrm{~Hz}\right) \delta_{\mathrm{C}} 172.4,140.5,128.7,128.6,128.2,127.3,126.6,125.6,124.8,123.1$, 120.6, 120.0, 115.5, 86.3, 84.3, 68.8, 54.4, 34.9, 29.9, 28.8, 21.3 ppm; Ms m/e (relative intensity) $375\left(\mathrm{M}^{+}\right.$- 57, 14.01), 319 (67.32), 275 (33.99), 257 (9.86), 206 (22.32), 144 (26.39), 84 (42.56), 57 (100.00); HRMS for $\mathrm{C}_{26} \mathrm{H}_{27} \mathrm{NO}_{5}$ : 433.1889; found: 433.1889.

\section{Methyl (+)-2-hydroxyl-2-[(N-}

tbutoxylcarbonyl)-3-indole]-5-

\section{tbutyldimethylsilyloxyl-3-yn-pentyrate (4g):}

$\mathrm{Zn}(\mathrm{OTf})_{2}(0.2$ eq.) as additive, $(1 S, 2 S)-\mathbf{1}$ as ligand: Isolated in $67 \%$ yield and $81 \%$ ee (the reaction scale: Alkyne $3 \mathbf{c}$ is $2.5 \mathrm{mmol}$, the $\alpha$-keto ester $\mathbf{2 c}$ is $0.5 \mathrm{mmol}$ ) as determined by HPLC analysis (Chiralcel AD, hexane / $i-\mathrm{PrOH}=8 / 2,254 \mathrm{~nm}$ ) $\mathrm{t}_{\mathrm{r}} 8.13$ (minor); 9.20 (major); $[\alpha]_{\mathrm{D}}{ }^{20}=+18.9\left(c, 1.42, \mathrm{CHCl}_{3}\right)$; IR (neat) 3487, 2931, 2858, 1739, 1454, 1374, $1257,1234,1158,1091,1061,837,780,748,667 \mathrm{~cm}^{-1} ;{ }^{1} \mathrm{H}$ NMR $\left(\mathrm{CDCl}_{3}\right) \delta_{\mathrm{H}} 8.10(\mathrm{~d}, J=$ $7.5 \mathrm{~Hz}, 1 \mathrm{H}), 7.80(\mathrm{~s}, 1 \mathrm{H}), 7.60(\mathrm{~d}, J=7.5 \mathrm{~Hz}, 1 \mathrm{H}), 7.30$ - 7.20 (m, $2 \mathrm{H}), 4.40(\mathrm{~s}, 2 \mathrm{H})$, 4.05 (br, $1 \mathrm{H}), 3.75(\mathrm{~s}, 3 \mathrm{H}), 1.60(\mathrm{~s}, 9 \mathrm{H}), 0.85$ (s, $9 \mathrm{H}), 0.08(\mathrm{~s}, 6 \mathrm{H}) \mathrm{ppm} ;{ }^{13} \mathrm{C}$ NMR $\left(\mathrm{CDCl}_{3}, 75 \mathrm{~Hz}\right) \delta_{\mathrm{C}} 171.8,149.4,136.1,127.0,125.4,124.7,122.9,120.4,119.2,115.3$, 84.5, 84.1, 81.9, 68.6, 54.2, 51.7, 29.7, 28.2, 25.8, -5.2 ppm; Ms m/e (relative intensity) 415 ( $\mathrm{M}^{+}$- 58, 17.06), 359 (100.00), 329 (18.78), 315 (45.27), 299 (28.66), 269 (17.01), 198 (14.24), 154 (51.86), 57 (88.89); HRMS for $\mathrm{C}_{25} \mathrm{H}_{35} \mathrm{NSiO}_{6}$ : 473.2192; found: 473.2213 .

\section{Ethyl (-)-(R)-2-hydroxyl-2-methyl-4-phenyl-3-yn-butyrate (4h):}

$\mathrm{Zn}(\mathrm{OTf})_{2}(0.2$ eq.) as additive: Isolated in $11 \%$ yield and $92 \%$ ee (the reaction scale: Alkyne $3 \mathbf{a}$ is $1.5 \mathrm{mmol}$, the $\alpha$-keto ester $\mathbf{2 d}$ is $0.5 \mathrm{mmol}$ ) as determined by HPLC analysis (Chiralcel OD, $i$-PrOH $/$ hexane $=97 / 3,254 \mathrm{~nm}$ ) $\mathrm{t}_{\mathrm{r}} 20.647$ (minor), 23.900 (major); $[\alpha]_{\mathrm{D}}{ }^{20}=-15.3\left(c, 0.38, \mathrm{CHCl}_{3}\right)$; IR (neat) 3484, 2988, 2940, 2238, 1739, 1599, 1491, 1445, $1251,1150,1126,1017,758,692 \mathrm{~cm}^{-1} ;{ }^{1} \mathrm{H}$ NMR $\left(\mathrm{CDCl}_{3}\right) \delta_{\mathrm{H}}$ 7.5-7.45 (m, 2H), 7.35-7.25 (m, 3H), 4.35 (q, $J=7.3 \mathrm{~Hz}, 2 \mathrm{H}), 3.75$ (br, $1 \mathrm{H}), 1.80$ (s, 3H), 1.35 (t, $J=7.3 \mathrm{~Hz}, 3 \mathrm{H})$ ppm; ${ }^{13} \mathrm{C} \mathrm{NMR}\left(\mathrm{CDCl}_{3}, 75 \mathrm{~Hz}\right) \delta_{\mathrm{c}} 173.0,132.1,128.9,128.5,122.2,88.7,84.1,68.5,63.2,27.4$, $14.3 \mathrm{ppm}$; Ms m/e (relative intensity) $218\left(\mathrm{M}^{+}, 1.88\right), 210$ (60.25), 173 (9.06), 145 
(100.00), 129 (15.32), 115 (4.77), 43 (38.15); Anal. Cald for $\mathrm{C}_{13} \mathrm{H}_{14} \mathrm{O}_{3}$ : C, 71.54\%; H, 6.47\%. Foumd: C, $71.63 \%$; H, 6.49\%.

Stereochemistry determination: In order to determine the absolute configuration, adduct $4 \mathrm{~h}$ was then hydrogenated with Pd-C in the pressure of 4 atm hydrogen to afford $\alpha$-hydroxyl ester 6. Comparing the optical rotation of the synthesized $6\left(-26, \mathrm{c}=0.26, \mathrm{CHCl}_{3}\right)$ and the rotation data of ethyl (S)-2-hydroxy-2-methyl-4-phenyl-butyrate, which has been reported as $+29.6\left(\mathrm{c}=2.4, \mathrm{CHCl}_{3}\right)$, the absolute stereochemistry of the tertiary asymmetric alcohol carbon in $\mathbf{4 h}$ was assigned as $R$ configuration.

\section{(+)-2-hydroxyl-2-phenylethynyl-3,3-dimethyl-pentalactone (4i):}

$\mathrm{Zn}(\mathrm{OTf})_{2}(0.2$ eq. $)$ as additive, $(1 S, 2 S)-1$ as ligand: Isolated in $95 \%$ yield and $93.5 \%$ ee (the reaction scale: Alkyne $3 \mathrm{a}$ is $1.5 \mathrm{mmol}$, the $\alpha$-keto ester $2 \mathrm{e}$ is $0.5 \mathrm{mmol}$ ) as determined by HPLC analysis (Chiralcel AD, hexane / $i-\mathrm{PrOH}=9 / 1,254 \mathrm{~nm}$ ) $\mathrm{t}_{\mathrm{r}} 11.647$ (major), 15.590 (minor); m.p. $66-70^{\circ} \mathrm{C} ;[\alpha]_{\mathrm{D}}{ }^{20}=+8.89\left(c, 3.92, \mathrm{CHCl}_{3}\right)$; IR (neat) 3412, 2966, 2930, $2240,1764,1492,1385,1367,1266,1177,1086,1052,1014,997,847,757,693 \mathrm{~cm}^{-1} ;{ }^{1} \mathrm{H}$ $\operatorname{NMR}\left(\mathrm{CDCl}_{3}, 300 \mathrm{MHz}\right) \delta_{\mathrm{H}} 7.65(\mathrm{~m}, 2 \mathrm{H}), 7.55(\mathrm{~m}, 3 \mathrm{H}), 4.35\left(\mathrm{dd}, J_{1}=25.4 \mathrm{~Hz}, J_{2}=8.7\right.$ $\mathrm{Hz}, 2 \mathrm{H}), 4.05(\mathrm{br}, 1 \mathrm{H}), 1.60(\mathrm{~s}, 3 \mathrm{H}), 1.45$ (s, 3H) ppm; ${ }^{13} \mathrm{C} \mathrm{NMR}\left(\mathrm{CDCl}_{3}, 75 \mathrm{~Hz}\right) \delta_{\mathrm{C}} 174.9$, 131.9, 129.2, 128.3, 121.2, 89.4, 82.8, 76.8, 74.7, 44.6, 20.5, 19.6 ppm; Ms m/e (relative intensity) 230 ( $\left.\mathrm{M}^{+}, 6.72\right), 202$ (4.21), 186 (13.01), 175 (16.99), 171 (100.00), 157 (13.61), 143 (24.90), 129 (46.36), 115 (11.47); HRMS for $\mathrm{C}_{14} \mathrm{H}_{14} \mathrm{O}_{3}$ : 230.0941; found: 230.0942.

\section{(-)-2-hydroxyl-2-(4-phenyl-1-butynyl)-3,3-dimethyl-pentalactone (4j):}

$\mathrm{Zn}(\mathrm{OTf})_{2}(0.2$ eq.) as additive, $(1 S, 2 S)-\mathbf{1}$ as ligand: Isolated in $93 \%$ yield and $94 \%$ ee (the reaction scale: Alkyne $\mathbf{3 b}$ is $1.5 \mathrm{mmol}$, the $\alpha$-keto ester $\mathbf{2 e}$ is $0.5 \mathrm{mmol}$ ) as determined by HPLC analysis (Chiralcel AD, hexane / $i-\mathrm{PrOH}=95 / 5,254 \mathrm{~nm}$ ) $\mathrm{t}_{\mathrm{r}} 22.21$ (minor), 25.20 (major); $[\alpha]_{\mathrm{D}}{ }^{20}=-17.5\left(c, 0.45, \mathrm{CHCl}_{3}\right)$; IR (neat) 3438, 2980, 2232, 1769, 1265, 1223, 1089, 1003, 697, $470 \mathrm{~cm}^{-1} ;{ }^{1} \mathrm{H}$ NMR $\left(\mathrm{CDCl}_{3}, 300 \mathrm{MHz}\right) \delta_{\mathrm{H}} 7.30-7.15(\mathrm{~m}, 5 \mathrm{H}), 3.95$ $\left(\mathrm{dd}, J_{1}=16.5 \mathrm{~Hz}, J_{2}=8.5 \mathrm{~Hz}, 2 \mathrm{H}\right), 3.20(\mathrm{br}, 1 \mathrm{H}), 2.79(\mathrm{t}, J=7.3 \mathrm{~Hz}, 2 \mathrm{H}), 2.5(\mathrm{t}, J=7.3 \mathrm{~Hz}$, 2H), 1.05 (s, 3H), 1.04 (s, 3H) ppm; ${ }^{13} \mathrm{C} \mathrm{NMR}\left(\mathrm{CDCl}_{3}, 75 \mathrm{~Hz}\right) \delta_{\mathrm{C}} 175.0,140.0,128.4$, 126.5, 90.0, 76.6, 75.5, 74.3, 44.1, 34.3, 20.8, 20.3, 19.4 ppm; Ms m/e (relative intensity) 
$214\left(\mathrm{M}^{+}\right.$- 44, 1.22), 199 (29.62), 181 (9.90), 157 (13.33), 129 (16.55), 91 (100.00), 69 (43.13); HRMS for $\mathrm{C}_{16} \mathrm{H}_{18} \mathrm{O}_{3}$ : 258.1246; found : 258.1251 .

Ethyl (-)-2-hydroxyl-2-methyl-4-phenyl-butyrate (6):

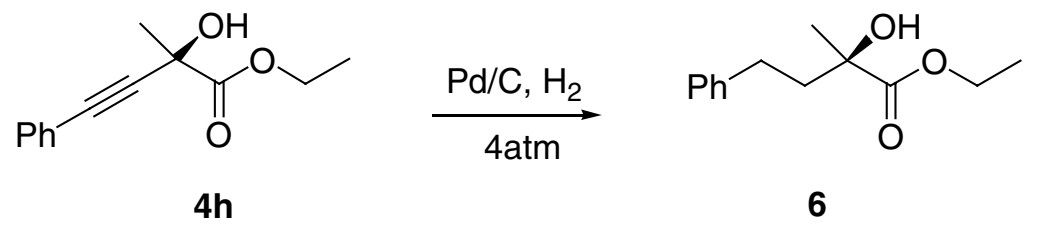

A solution of $\mathbf{4 h}(10 \mathrm{mg})$ in ethyl acetate $(8 \mathrm{~mL})$ was treated with $10 \%$ Palladium hydroxide on carbon ( $3 \mathrm{mg}$ ), and $4 \mathrm{~atm}$ of hydrogen in a Parr apparatus. After $24 \mathrm{~h}$, the reaction mixture was filtered through Celite and concentrated to obtain crude material, which was purified through flash chromatograph (petroleum: ethylacetate $=10: 1$ ) to afford colorless oil $6(6 \mathrm{mg}, 59 \%):[\alpha]_{\mathrm{D}}{ }^{20}=-26\left(c, 0.26, \mathrm{CHCl}_{3}\right)$; IR (neat) 3522, 3023, 2981, 2928, 2856, 1729, 1604, 1498, 1455, 1375, 1251, 1191, 1118, 1070, 1022, 749, $700 \mathrm{~cm}^{-1}$; ${ }^{1} \mathrm{H} \mathrm{NMR}\left(\mathrm{CDCl}_{3}\right) \delta_{\mathrm{H}} 7.30(\mathrm{~m}, 2 \mathrm{H}), 7.20(\mathrm{~m}, 3 \mathrm{H}), 4.22(\mathrm{q}, J=7.0 \mathrm{~Hz}, 2 \mathrm{H}), 3.35(\mathrm{br}, 1 \mathrm{H})$, $2.80(\mathrm{~m}, 1 \mathrm{H}), 2.45(\mathrm{~m}, 1 \mathrm{H}), 2.05(\mathrm{~m}, 2 \mathrm{H}), 1.45(\mathrm{~s}, 3 \mathrm{H}), 1.30(\mathrm{t}, J=7.0 \mathrm{~Hz}, 3 \mathrm{H}) \mathrm{ppm} ;{ }^{13} \mathrm{C}$ $\mathrm{NMR}\left(\mathrm{CDCl}_{3}, 75 \mathrm{~Hz}\right) \delta_{\mathrm{C}} 141.6,128.4,125.9,74.1,61.9,41.7,30.1,26.3,14.2 \mathrm{ppm}$; Ms m/e (relative intensity) $222\left(\mathrm{M}^{+}, 0.40\right), 205$ (2.61), 183 (0.60), 149 (23.18), 131 (15.81), 118 (37.88), 105 (9.50), 91 (100.00), 43 (16.08); Anal. calcd. for $\mathrm{C}_{13} \mathrm{H}_{18} \mathrm{O}_{3}: \mathrm{C}, 70.24 \%$; $\mathrm{H}$, $8.16 \%$. found: $\mathrm{C}, 57.82 \% ; \mathrm{H}, 70.29 \% ; \mathrm{N}, 8.13 \%$. 